\title{
Teaching Musical Expression through Visualization of Musical Cues
}

\author{
P.J. Peiris-Perera* \\ *Dept. of Western Music, University of Visual and Performing Arts, Colombo, Sri Lanka.
}

\begin{abstract}
Many musicians agree that some music recital performances seem more academic than artistic. These performances fail to convey the character of the music. An important aspect of teaching music is to develop abilities of students in musical expression. Performance studies have shown that music teachers and students are often unaware of tools they could utilize to develop musical expression. In order to find a solution to this problem, this paper based its groundwork on Patrick Juslin's GERMS model. The GERMS model provides a mechanism to conceptualize musical expressions as a multi-dimensional phenomenon. In this work we introduce an innovative visualization model named EmoGraph that can be used to visualize the emotional expression aspects of music scores using the GERMS model in order to help the students grasp the cues of musical expressions. This visualization tool helps to capture the cues for emotional expressions in a musical score for the purpose of using it as a teaching tool to teach musical expressions. This work also introduces an experimental setup that can be used to evaluate the effectiveness of the proposed teaching tool at an undergraduate academic environment.
\end{abstract}

Keywords: Musical-Expressions; Emotions; GERMS; Pianists; Musical-Performance

\section{Introduction}

How often have we heard performances that were played with absolute faithfulness to the score but which failed to convey the character of the music? A study conducted by Karlssohn and Juslin on teachers' opinions about musical expression showed that teachers thought that musical expression was one of the most important elements in music making and teaching (Karlsson \& Juslin, 2008). However, many teachers do not know about the tools that could aid in the development of musical expression. This work explores and examines recent studies in music and emotion in order to develop successful strategies that can be used to teach musical expression. This work focuses on just one aspect of musical expression; that is emotional expression. This aspect has been noted as the least explored in terms of research and also one of the hardest elements to teach.

The term "musical expression" encompasses many different facets, meanings, and definitions. In his study, the "Five Facets of Musical Expression: A Psychologist's Perspective on Music Performance,” Patrick Juslin constructed the "GERMS” model, which is based on a wide array of previous research from multiple sources on this subject (Juslin, 2003). This model can be used as a tool to analyse musical expression in performances as well as a teaching model. He proposed five facets for this model, which he believes to be a multi-dimensional approach to enhancing musical expression. The five facets of this GERMS model are: Generative rules (G), Emotional expression (E), Rhythmic properties (R), Motion principles (M), and Stylistic unexpectedness (S). For the purpose of this paper, This work examines only the application of the "Ecomponent" of the GERMS model, which is "Emotional Expression."

The "Emotional Expression" component has been noted as the most challenging and complicated aspect to establish data (Meyer, 1957). In addition, there are different uses of the word expression in the literature, and sometimes it has been used to refer to the emotional qualities of music by listeners (Davies, 1994). Nonetheless it is accepted that these two senses of the word are related (Davies, 1994). Thus Juslin and Persson 
defines "Emotional Expression” as the “...use of systematic variations in performance parameters (such as timing, dynamics, timbre) to convey emotions to listeners (Juslin \& Persson, 2002).”

Sometimes things that are most important to learn are the most difficult to teach(Karlssohn \& Juslin, 2008). Musical expression should be taught from the beginning, but emotional expression can be hard to teach. This does not mean that the teacher should completely overlook the possibilities of encouraging emotional expression; rather, the teacher will need to incorporate it whenever possible. Elementary-level repertoire often lacks the properties which contribute to emotional expression due to limitations in scope (for example, pieces which are extremely short). On the other hand, intermediate literature can provide a good foundation for developing the emotional expression component, because the pieces become much more elaborate in terms of their content, harmonic language, and dynamic range, where opportunities to express one's self abound. In this work we recommend and use intermediate repertoire for teaching emotional expression to students.

Emotional response and musical expectation are closely linked (Meyer, 1957). The theory here is that emotion is experienced when a musical outcome is different from that expected by the listener. For example, when the expectation is a perfect cadence but the actual outcome is an interrupted cadence, this evokes an emotional response in the listener and it has been found that listeners' familiarity with pieces of music does not affect this emotional response. Emotional expression involves the use of cues which are interpreted by both the performer and the listener. These cues might be tempo, loudness, timing, timbre, etc., and they are used by the performer to express emotions such as anger, sadness, and love/tenderness (Juslin, 2003). There is evidence to suggest that happiness is expressed through "fast tempo, high sound level, staccato articulation, large articulation variability, fast tone attacks, and bright timbre" (Juslin, 2003) whilst sadness is expressed through "slow tempo, low sound level, legato articulation, small articulation variability, slow tone attacks, and dull timbre” (Gabrielsson \& Juslin, 1996).

Using the Emotional expression (E) component of the GERMS model it is possible to quantify the level of emotional expression of a musical score. Multi-dimensional nature of the quantification of the musical scores requires a visualisation that can display multivariate data. In this work we suggest the use of a standard radar-chart to visualise the musical expression as a teaching aid named EmoGraph, which is a six dimensional graph that combines the tempo, temp-variability, sound sound-variability, articulation and articulation-variability. Figure 1 shows the basic visualisation template suggested in this paper. Basic colours Red, Green and Blue are used to separate the tempo, sound and the articulation as the background. The colour of the resultant EmoGraph will combine the Red, Green and Blue intensity based on the values required to represent the respective musical score similar to what is normally done on a standard heat map visualization. Table 1 shows the suggested mapping for the visualization and Figure 2 show samples of visualizations for the basic emotional expressions for Happy, Sad, Tender, and Anger based on the suggested visualization scheme in this paper. 


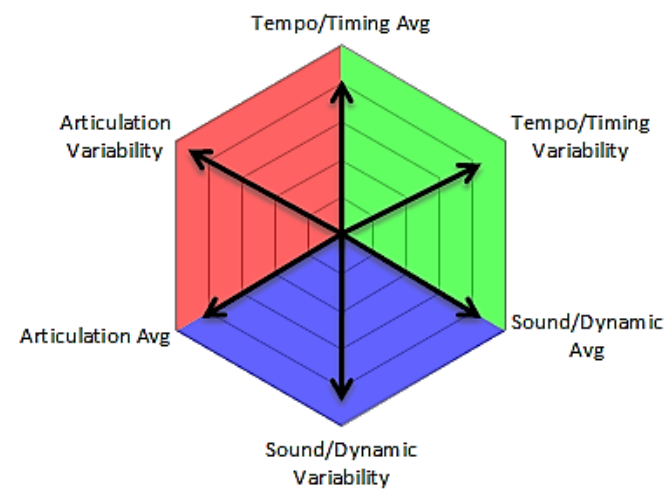

Figure 1 Basic Musical Score Visualization (EmoGraph) Template

Table 1 Mapping of Musical Cues for Visualization

\begin{tabular}{|c|c|c|c|c|c|}
\hline \multirow[b]{2}{*}{ Musical Cue } & \multirow{2}{*}{$\begin{array}{l}\text { Nominal Scale Categories for Musical } \\
\text { Cues }\end{array}$} & \multirow{2}{*}{$\begin{array}{l}\text { Value } \\
\text { Range }\end{array}$} & \multirow{2}{*}{$\begin{array}{l}\text { Vis. } \\
\text { Step } \\
\text { Wt. }\end{array}$} & \multicolumn{2}{|c|}{ Example Computation For “Happy” } \\
\hline & & & & $\begin{array}{c}\text { Musical Cue } \\
\text { Value }\end{array}$ & $\begin{array}{c}\text { Visualization } \\
\text { Weight }\end{array}$ \\
\hline Tempo-Average & $\begin{array}{l}\text { V.slow / slow / med.slow/ med.fast / fast / } \\
\text { V.fast }\end{array}$ & $1-6$ & 0.167 & (Fast) & 0.83 \\
\hline Tempo-Variability & Low / medium / high & $1-3$ & 0.333 & (low) & 0.33 \\
\hline Sound-Average & $\mathrm{ppp} / \mathrm{pp} / \mathrm{p} / \mathrm{mp} / \mathrm{mf} / \mathrm{f} / \mathrm{ff} / \mathrm{fff}$ & $1-8$ & 0.125 & (ff) & 0.88 \\
\hline Sound- Variability & Low/ medium / high & $1-3$ & 0.333 & (low) & 0.33 \\
\hline $\begin{array}{l}\text { Articulation- } \\
\text { Average }\end{array}$ & Legato / strong / accents / portato / staccato & $1-5$ & 0.200 & (staccato) & 1.00 \\
\hline $\begin{array}{l}\text { Articulation- } \\
\text { Variability }\end{array}$ & Low / medium / high & $1-3$ & 0.333 & (high) & 1.00 \\
\hline & \multicolumn{5}{|c|}{ Visualization Weight $=$ Musical Cue Value $\times \frac{1}{\text { Max Musical Cue Value }}$} \\
\hline
\end{tabular}
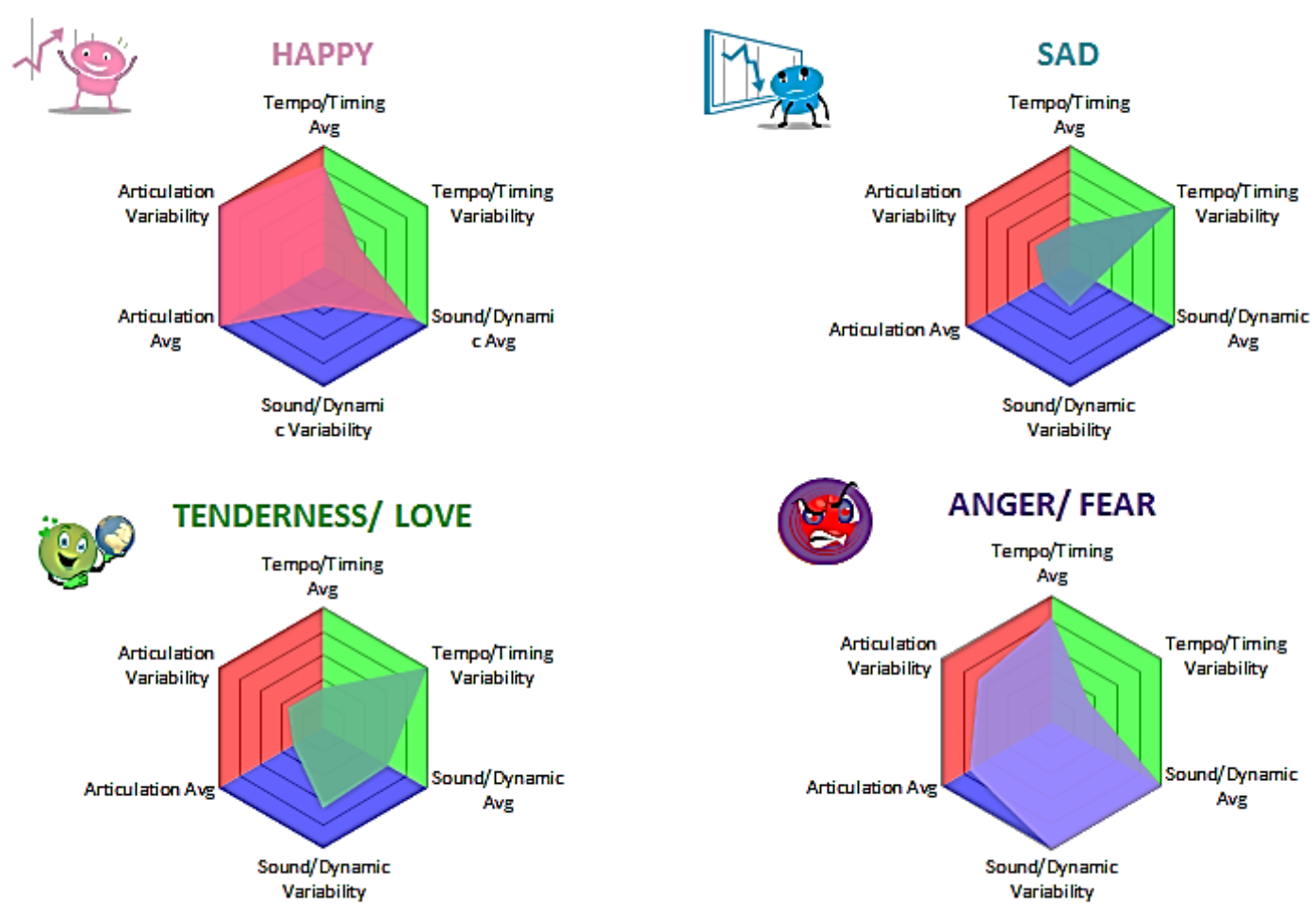

Figure 2 EmoGraph Visualization of Expressive Cues 
In Table 1, the range of values for each attribute and the value selections for each emotion is based on the suggestions in the GERMS model (Juslin, 2003). Last two columns of Table 1 includes the computation of the visualisation mapping for the emotion "Happy" as an example. Note that the clipart images used in conjunction with the corresponding visualization is intended only for the purpose of enhancing the emotional expression and can be replaced with any other appropriate artwork.

\section{Methodology}

The purpose of this study is to introduce and examine the effects of utilizing the computer-based visual model as a teaching aid to increase musical expression in piano performances. Subjects are undergraduate piano majors and minors at the Department of Western Music of the University of Visual and Performing Arts, Colombo, Sri Lanka, enrolled for class piano study. Subjects were asked to briefly review a piece that has been previously studied. We identified two sections that were musically challenging in the piece where students were more likely to play without much musical expression. After recording a play-through of the piece they rated the level of musical expression on a scale of 1 to 5 (with 1 indicative of "no expression" and 5 representative of "Highest level of expression") when performing each of the passages. Approximately one week following, one group received instructions on the "visualization" to develop musical expression to the piece while the control group did not, instead they practiced and recorded the piece. The group with EmoGraph "visualization" were allowed to practice for several minutes. Following this session they recorded a play-through of the piece. Figure 3 shows a sample visualization for one musical score used in the study by modern composer Emma Lou Diemer called "Anger". The colour of the graph indicates more Red and Blue and also the values indicate more sound and articulation in parts of the score that were selected for emotional expressions.

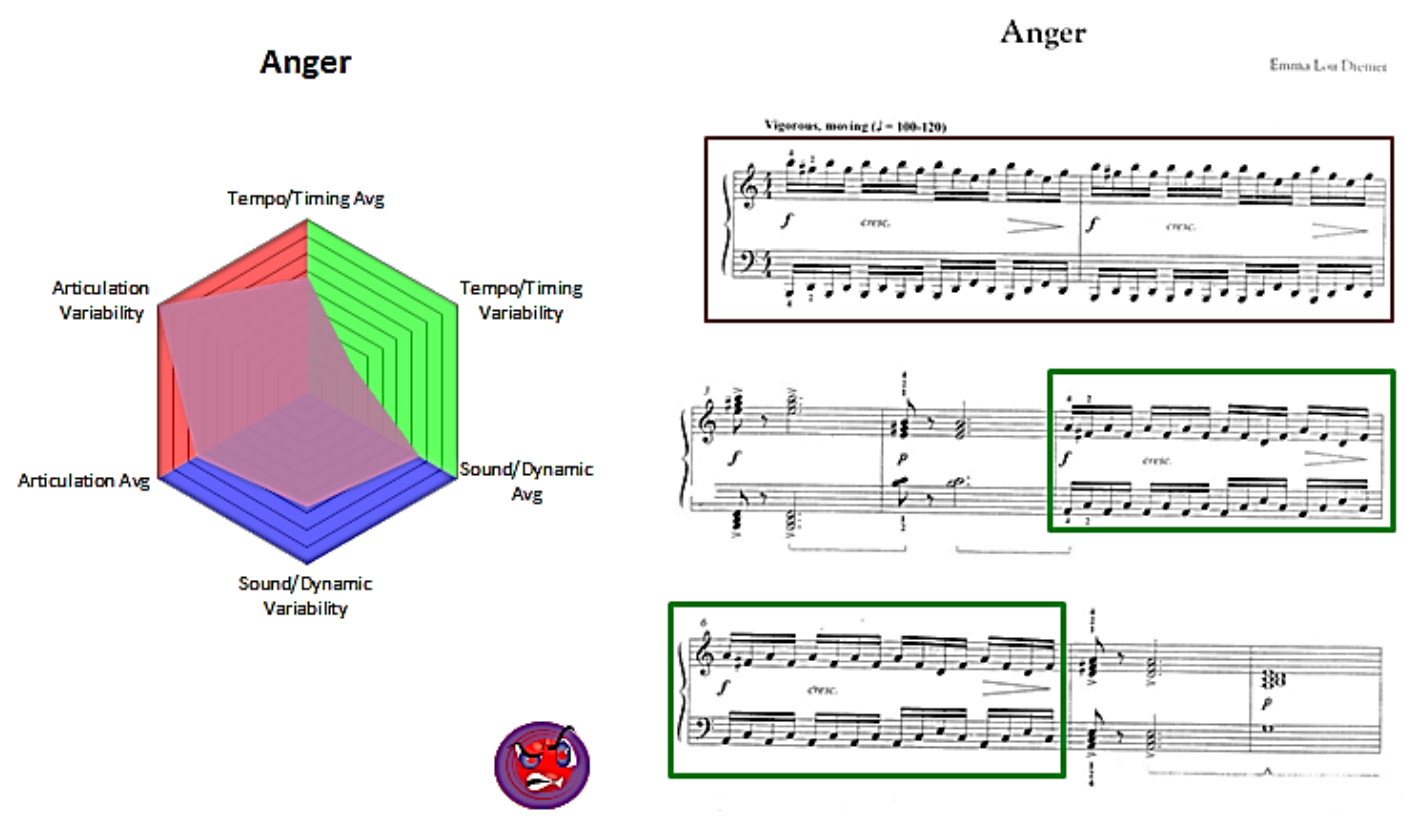

Figure 3 Example Visualization for “Anger” by Emma Lou Diemer 
The selected parts are highlighted in the score. Note the difference between this visualization and the visualization for the emotion anger given in Figure 2. Figure 2 shows the visualization for the particular emotion anger and Figure 3 shows the visualization for the musical score "Anger."

Following the process of performing in the class room for the study, audio recordings were extracted, compiled, and randomly ordered on a CD. Five independent adequately qualified judges were requested to listen to the recordings of the pieces and rate each performance, based on an evaluation rubric taking into account performers' note, rhythm accuracy, and musicality. A basic statistical comparison with the control group is sufficient to show the effectiveness of the EmoGraphs as a teaching aid to teach musical expression.

\section{Results and Discussion}

Musical expression is a very important aspect in music. Teaching musical expression is a challenge to most teachers based on available studies and is also evident when witnessing most university music student performances. Most university music majors also choose teaching music as their future careers. It is vital that teachers, engaged in teaching university students utilize all available tools to teach musical expression so that the future generation of music teachers are exposed to these techniques. Emotional expression is a major component in musical expression. This work introduces a novel visualization technique called EmoGraph based on the GERMS model to extract the emotional cues in a musical score to enable a student to easily identify and relate to the expected emotional expressions in a repertoire. The teaching tool will prompt, guide and help the student to identify the cues but still allow the freedom to express emotions based on their own interpretation of the score.

A basic experimental study is also outlined to test the validity of the proposed tool. Subjects' performance evaluation for musical expression is based on human judgements. In practice it is very hard to collect evaluations from experts in the field of musical judgement on a voluntary basis, thus the data collection for the study is still continuing with most of the data collected. Preliminary results indicate a positive impact to the use of the EmoGraphs. To make a scientifically conclusive judgment all results should be compiled and statistically analysed.

As a future study it is suggested to do the subject performance evaluation for musical expression based on software analysis and visualization of subjects' performances, on musical tracks using a software such as "Reason" (Propellerhead, 2015). Most of the modern piano keyboards used for teaching university class piano courses allow the recording of performances as MIDI tracks and currently, the available audio recording and editing software such as "Reason," allows the automated capture and visualization of emotional cues such as sound, tempo and articulation. This cannot completely eliminate the human judges but can reduce the involvement. The use of computer tools in conjunction with the voluntary human judges could address the issue of data collection faced by the current work.

The framework introduced in this study can also be used appropriately for the other aspects of the GERMS model. This could lead to the introduction of other visualizations similar to the EmoGraph that can aid in teaching other aspects of musical expressions that are vital to produce a properly interpreted, outstanding musical performance that is not a mere academic mechanical reproduction of the musical score. 


\section{Conclusion}

An important aspect of teaching music is to develop musical expression though it is considered hard in many studies. This work attempts to address the difficulty of teaching musical expression to students using a visualization based on the GERMS model. This visualization captures the musical cues in a musical score for emotional expression as an EmoGraph that can be used as a teaching aid. A six dimensional radarchart combined with a heat map is used as the basis for the EmoGraph visualization. An experimental framework that can be used to evaluate the proposed EmoGraph based teaching of musical expression is also suggested in this work.

\section{References}

Davies, S. (1994). Musical Meaning and Expression. CA, USA.: Cornell University Press.

Gabrielsson, A., \& Juslin, P. N. (1996). Emotional Expression in Music. Handbook of Affective Sciences 503-534. New York, NY, USA: Oxford University Press.

Juslin, P. N. (2003). Five Facets of Musical Expression: A Psychologist's Perspective on Music Performance. Psychology of Music, 31, 273-302.

Juslin, P. N., \& Persson, R. (2002). Emotional Communication. In Science \& Psychology of Music Performance: Creative Strategies for Teaching and Learning. New York, NY, USA: Oxford University Press.

Karlssohn, J., \& Juslin, P. N. (2008). Musical Expression: An Observational Study in Instrumental Teaching. Psychology of Music, (36), 309-337.

Karlsson, J., \& Juslin, P. N. (2008). Musical Expression: An Observational Study of Instrumental Teaching. Psychology of Music, 36: 3, 309-337.

Laukka, L. (2004). A Report From Music Conservatoires. Music Education Research, 6, 45-56.

Marcel, T. (1999). Pedagogical Concepts and Practices for Teaching Musical Expressiveness (D.M.A.). Ohio State University, Ohio.

Meyer, L. (1957). Emotion and Meaning in Music. Chicago, IL, USA.: University of Chicago Press.

Parmer, C. (1996). Anatomy of a performance: Sources of musical expression. Music Perception (Vol. 13, pp. 433-454).

Parncutt, R., \& McPherson, G. (2002). The Science \& Psychology of Music Performance: Creative Strategies for Teaching and Learning. New York, NY, USA: Oxford University Press.

Propellerhead. (2015). Reason. Retrieved from https://www.propellerheads.se/reason

Radocy, R., \& Boyle, D. (2003). Psychological Foundations of Musical Behaviour. Chicago, IL, USA.: Charles Thomas Pub. 